\title{
Periodization in Team Sport Games - A Review of Current Knowledge and Modern Trends in Competitive Sports
}

\author{
by

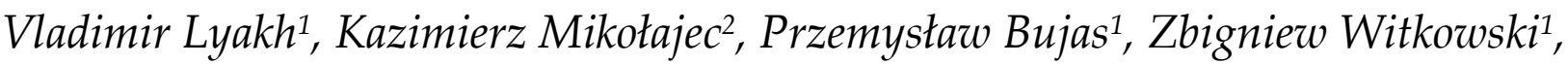 \\ Tomasz Zajaci3, Ryszard Litkowycz'2, Damian Banyś2
}

The main goal of this study was to present a review of current knowledge and modern trends in periodization of the training process in team sports. The research objectives were: an analysis of various aspects of periodization of the annual training cycle for elite athletes practicing team sport games, an attempt to determine both the examined and unexamined issues related with periodization of training as well as to indicate directions for further research, and finally, presentation of different training loads and competitions in micro-, meso- and macrocycles. The research consisted of the analysis and generalization of the bibliography, methods of monitoring training and competition loads of the Polish national U17 female soccer team in the seasons 2011/2012 and 2012/2013, as well as of the female basketball division one club in the season 2014/2015. Findings of the present study indicate resolved as well as unresolved aspects of annual training cycle periodization in team sport games and provide information on the types of training and competitive workload planning in micro-, meso- and macrocycles.

Key words: team sport games, periodization, training loads, training cycles.

\section{Introduction}

Specialists from many countries agree that sport preparation is a holistic and multiannual process, during which the athlete aims to achieve his or her top performance in order to contend in most prestigious competitions. The achievement of this goal is impossible when attempted without the consideration of the fundamental principles of periodization, which is understood as structuring of the training process and competition participation into various phases, periods and cycles. The concept of periodization in sports and academic research is associated with the name of a prominent specialist, Matwiejew, who, in 1964, published his work "The problem of sport training periodization". The author proved that proper and periodic alteration of the structure and content of the training process in its successive cycles was the basis of rational periodization for elite athletes, as it aimed at achieving top performance before participating in the main competitions (World Championships, European Championships, Olympic Games, etc.).

Fundamental works on periodization of sports training have been published by Matwiejew (1977, 1987, 1991, 1997, 1999) and other Russian (Liszczenko, 1997, 2014; Ozolin, 1970; Suslow, 1995, 2002; Suslov and Fillin, 1998) and Ukrainian specialists (Keller, 1995; Platonov, 1986, 1997, 1998, 2004, 2008, 2013). Although scientists from countries of the former Soviet Union (Bondarczuk, 2000, 2005, 2010; Issurin, 2010, 2010a, 2008; Verhoshansky, 1985, 1998,

\footnotetext{
1 - Department of Theory of Sport and Kinesiology, Academy of Physical Education in Cracow.

2 - Department of Team Sport Games, Academy of Physical Education in Katowice.

3 - Human Performance Laboratory, Academy of Physical Education in Katowice
} 
1998a, 2005) as well as other countries: Australia (Haff, 2004; Haff and Haff, 2012), Germany (Harre, 1982; Pfeifer and Harre, 1982), Canada (Bompa, 1994, 1999, 2002, 2006; Bompa et al., 2009), USA (Kraemer, 2004; Maglischo, 2003; O'Bryand, 2004; Stone, 2004; Stone et al., 2007), Hungary (Nadori and Granek, 1989; Malacko, 2004), also significantly enriched this topic.

An especially valuable contribution to the development of the theory and practice of sport training periodization was made by a Ukrainian specialist - Platonov (2013). He not only presented the topic of periodization using an enormous amount of theoretical and empirical material supported by numerous examples, but also conducted most complex analysis of all other known concepts (models) of annual and multiannual periodization of training for athletes practicing various sport disciplines.

The research objectives were to analyze various aspects of periodization of the annual training cycle for elite athletes practicing different team sport games, determine both the examined and unexamined issues related to periodization of training as well as to indicate directions for further research, and finally, present different training loads and competitions in micro-, mesoand macrocycles.

\section{Material and Methods}

The present study based on the analysis and generalization of the bibliography, methods of monitoring training and competition loads of the Polish national U17 female soccer team in the seasons 2011/2012 and 2012/2013, as well as of the female basketball division one club in the season 2014/2015.

\section{Results and Discussion}

1. Resolved and unresolved aspects of annual training cycle periodization in team sport games.

The variety of microcycle structures of the preparatory and competitive periods are among the best examined aspects of periodization of annual training programs for elite players practicing team sport games. Fundamental works on this topic by Platonov (2013) demonstrated different types of microcycles for the competitive period in soccer players of leading European teams having one or two official games per week.
Bompa (2006) as well as Bompa and Haff (2010) described microcycles of the competitive phase for athletes considering not only one or two, but also three or four official games in a week. Similar data can also be found in works of Russian specialists which refer to (among others) basketball (Kostikowa and Wolosowicz, 1997), soccer (Godik, 1999) and volleyball (Bieliajew, 2000). A microcycle focused on the maintenance of specific fitness in team sport games has also been presented by Issurin (2010).

The three strategies of annual periodization listed by Platonov (2013) are an important theoretical accomplishment. The strategies differ both in terms of competitions, their number and structure and the content of the training process in various micro- and mesocycles.

According to the first strategy, the athletes as along with the team aim at achieving the best result in the main competition of the season. All other competitions are of secondary importance and participation in them serves as means of preparation and monitoring of the effectiveness of the training process. An effective realization of this strategy, as viewed by Platonov (2013), allows the players to achieve the best individual results in the main competition in $60-70 \%$ or in some situations in up to $90 \%$ of cases. The second strategy combines effective preparation for competition (in various disciplines) conducted over a long period of time (8-10 months) with the preparation for the participation in the main competition of the year. This strategy is characteristic for elite players. Its main problem is how to optimally combine workouts (in $4-7$ macrocycles) with the specific character of the games and their competition schedule in terms of annual periodization. The third strategy is, according to Platonov (2013), the most useful for athletes practicing team sport games. This strategy forces the players to participate in various competitions throughout the year including between 50 and 70 important events. The main concern of this strategy constitutes the optimal balance between work and rest as well as variability in training loads (volume and intensity).

Platonov's (2013) observations showed that usually none of the above mentioned strategies is used in its entirety. Depending on the 
competition schedule and the established priorities, players often combine different strategies. What draws the specialists' attention is the problem of annual training periodization for team players depending on the characteristics of their competition schedule, especially considering intensification of the athletes' activity in team games caused by increased professionalization and commercialization of sport (Liszczenko, 2014, 2014a; Mikolajec, et al., 2013; Platonov, 2013; Suslow, 2002). The annual competition schedule of most team sport games at the elite level does not allow for an optimal proportion between the number of training sessions and competitions to participate in. As a result, instead of planned adaptation, symptoms of de-adaptation can be observed in functioning of most important body systems (Platonov, 2013; Suslow, 2002).

Considering analysis of the unexamined or partially examined aspects of the annual periodization in team sport games, as mentioned before, empirically, it has not been proven how much time is required for various types of work in the annual training cycle of elite players involved in team sport games. As an example, in one of the works by Bompa (2006), referred to by Platonov (2013), is the author points out that the preparatory phase should last 6 months, the competitive phase 4 months and the transition phase 2 months. On the other hand, according to Platonov (2013), rational periodization of the annual training program in team sport games should include a preparatory phase of $8-12$ weeks, a competitive phase of $8-9$ months and a transition phase of $3-4$ weeks.

What also remains unexamined is the problem how to plan the development of various components of fitness in the preparatory and competitive phases of the annual program. For example, in the macrocycle structure, Bondarczuk (2005) distinguishes several stages of preparation i.e. physical, technical and game preparation. The concept of the development of various motor abilities (strength, speed, endurance, agility) has also been tackled by Issurin (2010) in his system of block periodization.

Bompa (2006), quoted by Platonov (2013), indicates that exercises aimed at the development of technical and tactical skills should take place in the first four months of the preparatory phase, while the development of motor abilities should be planned at the end of that period. Having this in mind, Platonov (2013) poses a question how to effectively improve technical and tactical skills without a proper level of physical fitness? In his opinion, such development of technical and tactical skills and physical preparation cannot be divided into separate, isolated stages, but should be combined into a holistic process, where the development of technique and tactics is conducted simultaneously with the development of crucial motor abilities.

Another issue unexamined so far is associated with the strategy of periodization of most significant motor abilities in team sport games. The range, intensity and complexity of workouts have not been established for athletes of different sports levels. It also remains unknown how to plan the workload for the athletes during the preparatory, competitive or transition phase.

So far, no compromise has been found with regard to which models of periodization fit best into the characteristics of modern team sport games: single-, double- or multicycle. For instance, it has been noticed that the competitive season in various sport disciplines often spreads to 35 - 40 weeks, therefore, a classic single-cycle annual model will not be able to ensure proper preparation for such long-term series of competitions (Platonov, 2013).

Our experiences gained through work with elite basketball teams, the Polish national basketball team (male) and national handball team (female, up to 17 years of age) grant us the basis to agree with Platonov's (2013) conclusions, which indicate that during the breaks between the games, chaotic (non-systemic and scientifically unjustified) planning of the training process can be often observed. Therefore, coaches and scientists urgently need to broaden their knowledge in this area.

2. Types of training and competitive workload planning in micro-, meso- and macrocycles.

As mentioned before, the problem of rational periodization of training sessions and competitions is described quite thoroughly both in scientific literature and training practice. This description is related to periodization at different levels: annual (macrocycle), during the preparatory, competitive and transition phase (in mesocycles) and in microcycles. 
For instance, the review of Platonov's (2013) works suggests that periodization of the annual preparatory cycle in today's soccer should adopt the following structure and content:

Preparatory phase (8-9 weeks)

Workload volume: 160 - 180 hours

Number of training sessions: 80 - 90

Workload direction:

- Multilateral, basic physical conditioning (motor abilities): 60 - 70 hours

-Versatile technical and tactical preparation: 50 - 60 hours

- Multilateral specific fitness: 40 - 50 hours.

Competitive phase (40 weeks)

Workload volume: 750 - 800 hours

Games: 45 - 55

Number of training sessions: 290 - 310

Workload direction:

- Baseline fitness (including a $30 \mathrm{~min}$ warm-up before competition: 90 - 110 hours)

- General conditioning: 140 - 150 hours

- Specific fitness: 170 - 220 hours

- Specific technical, tactical and psychological preparation: 250 - 300 hours

- Recovery and physiotherapy sessions: 60 80 hours.

Transition phase (3-4 weeks)

Workload volume: 20 - 25 hours

Number of training sessions: 9 - 12

Workload direction:

- Active rest

- Sustainment of aerobic endurance as well as power, speed and coordination abilities (jogging, swimming, rowing, general physical fitness exercises)

- Core stability exercises.

Periodization of the annual preparatory cycle in other sport disciplines is performed in a similar manner (Baechle et al., 2008; Beliajew, 2000; Platonov, 2013; Wathen et al., 2008).

The notion of the annual preparatory cycle periodization is based on the idea of integration of various aspects, which ensures a simultaneous development of motor abilities, technical and tactical skills, mental/personal features. It can be applied both in individual players and in relation to the whole team. Yet, works of some specialists from the United States are considered as exceptions (Baechle et al., 2008; Wathen et al., 2008). These authors associate the periodization of preparation in macrocycles, duration and content of the training sessions in mesocycles and microcycles with the principles regarding the development of various types of power.

As mentioned before, the literature related with sports training quite broadly describes the concepts of the duration, overall structure and content of various mesocycles (Beliajew, 2000; Godik, 1999; Platonov, 2013). Specialists claim that all components of the mesocycle should be dependent firstly on the principles of developing various components of fitness as well as on the annual schedule of main competitions. What also influences the mesostructure of the training process is the competition schedule, according to which, single-, double- or multicycle models of annual preparation are applied.

In case the competitive period lasts $8-10$ months, it is recommended to plan both competitive mesocycles (with a standard structure) and complex mesocycles of mixed character. Such a mesocycle should include the tasks of the following types of microcycles: preparatory, peaking, unloading and recovery (Platonov, 2013).

During the pre-competitive preparatory stage, the microcycles should resemble the model of the upcoming competitive regime. Several authors (Bieljaew, 2000; Godik, 1999; Platonov, 2013) claim that all technical-tactical tasks should be conducted under conditions imitating real game. The microcycle should consist of games and competitive preparation in $50-60 \%$ of its duration. From two to three weeks before the competition, the volume of the training workload should be lowered while maintaining the intensity of the competitive rigour. Moreover, from two to -four days before the competition, the intensity should also be decreased, allowing the players to fully recover and attain emotional readiness before the competition (Bejlajew, 2000; Bompa and Haff, 2010; Godik, 1999; Mikolajec, et al., 2013; Platonov, 2013).

Similarly to the preparatory period, the competitive period does not have its internal structure. The content and structure of its microcycles are dependent on the number of 
competitions in that period as well as on their principles.

In case there are two games within a week-long microcycle, the remainder of the tasks should be aimed at the players' recovery and preparation for the next game. According to Platonow (2013), the number of training sessions in such a cycle should be reduced to 7 and the total amount of the training workload as well as the volume of work should be reduced by $50 \%$. Moreover, suspension of resistance training with high loads $(80-100 \% 1 \mathrm{RM})$ should be considered. The workouts in such a microcycle should be organized as follows: 2 games $(3 \mathrm{~h}-$ game, $1 \mathrm{~h}-$ warm-up), training sessions $-8 \mathrm{~h}$, theoretical sessions - 3-3.5 h, recovery from 4 to $5 \mathrm{~h}$ (Platonov, 2013). Similar recommendations can be found in works of other authors (Bompa, 2006; Bompa and Haff, 2010).

With regard to the second type of training and competitive workload planning, the structure of the competitive period is much diversified. It is dependent on the players' schedule divided into several rounds: the participation in a number of games is followed by a several-week break. The morning practice after each game consists of lowintensity recovery exercises. A low-intensity tactical session is scheduled for the late afternoon on the day preceding each game. According to Bompa (2010), such a microcycle will allow the athlete to fully recover and to achieve optimal performance during the competition.

Considering the third type of training and competitive workload planning, the competitive period structures of the first and second types are combined. In the beginning, the games take place interspersed with even intervals (1 or 2 games per week); later the competitions take the shape of tournaments. The intervals between following stages are usually 2-3 week long. The training sessions are conducted with the aim of preparing the players for the next stage of the competition based on the earlier preparation in that period (Beliajew, 2000). Basic goals of these training sessions are similar to the ones already described, i.e. perfecting the technical-tactical skills, enhancing the level of preparation for the game, increasing mental resilience, sustaining physical fitness, creating circumstances for the players' recovery and continuation of theoretical sessions. Most of the training process is focused on preparing for the game, repeating the tactical schemes in various forms, specific exercises directed at sustaining the level of power-andspeed as well as coordination.

During the entire competitive period as well as the intervals between following rounds, the four-one, three-one, two-one microcycles are recommended. At the beginning of each microcycle, 1-2 days should consist of physical and mental recovery (Beliajew, 2000; Bompa and Haff, 2010).

During the transition period, most authors recommend active rest and substituting the specific exercises for general ones (crosscountry runs, cycling, hiking, swimming, gymnastics, acrobatics, other sport disciplines). It is also recommended to regularly perform power, flexibility, coordination and general endurance exercises. The workload should be reduced by $50 \%$ compared to the competitive and preparatory phases. The intensity of the performed exercises should be average or low. The number of training sessions within a week should oscillate around 56. However, body mass and body composition control should not be neglected.

\section{Conclusions}

The present literature review attempts to summarize and generalize the data regarding theoretical and practical issues around the concept of annual periodization in team sport games. Both examined and unexamined aspects of this problem have been addressed. At the same time, the perspectives for further research have been established. The review concentrates on the types of training and competition workloads in macro-, meso- and microcycles for elite athletes. Based on the conducted review of the strategies of rational annual periodization for team sport players, it was established that it should be organized as follows:

1) the preparatory phase, mandatory for all components of conditioning, should not be shorter than 8-12 weeks,

2) the competitive phase (8-9 months) should be directed at game performance effectiveness. The content and structure of the meso- and microcycles in this phase should take into account the limited co-dependency of training and competition. The most important parts of the training program include exercises 
aimed at technical and tactical skills, general and specific endurance, as well as coordination exercises. Theoretical and mental sessions should also be included. Of great importance is participation in games (exhibition, commercial or official) combined with procedures of recovery,

3) the transition phase (8-12 weeks) should be directed at full physical and mental recovery following the competitive period in the previous macrocycle. Mainly general exercises should be performed in this phase. Such exercises should ensure the sustainment of general fitness.

In case when fundamental principles of periodization are violated, the training process is chaotic and a tight competition schedule limits the athletes' possibilities in terms of preparation, rest and recovery after important tournaments. This situation often occurs in elite tennis players (Liszczenko, 2014). The people responsible for the organization and development of team games (at the national and international level) follow a policy which is oriented towards the commercialization of these sport disciplines. As a result, talented young athletes are often forced to end their careers in a particular team sport at an early age. A high number of competitions often causes overreaching, overtraining and increases the risk of injury.

In such cases, only athletes who limit their competitive activity (through the reduction of the number of tournaments and compliance with the norms established by the scholars in terms of the number of competitions per year) are able to maintain their health and extend their sports careers. The analysis of the literature associated with the annual training cycle of athletes presenting various performance levels shows that such aspects require further theoretical and experimental research.

\section{References}

Baechle T, Earle R. Essentials of Strength Training and Conditioning. Human Kinetics, Champaign IL; 2008

Beljaew A. Sport training periodization - Volleyball. Fizkultura, Moscow, 2000: 262-267

Bompa T. Theory and Methodology of Training. The key to Athletic Performance. Kendall/Hunt, Dubuque, IA; 1994

Bompa T. Periodization: Theory and Methodology of Training. Human Kinetics, Champaign IL; 1999

Bompa T. Total training for coaching team sports. Sport Books Publisher, Toronto; 2006

Bompa T, Carrera M. Periodization training for Sports: Science-Based Strenght and Conditioning Plans for 20 Sports. Human Kinetics, Champaign IL; 2005

Bompa T, Haff G. Periodization: theory and methodology of training. Human Kinetics, Champaign IL, 63-84; 2009

Bondarczuk A. Periodization of sports training. Olimpic Literature; 2000

Bondarczuk A. Periodization of sports training. Olimpic Literature; 2005

Godik M. Planning of sports training. Fizkultura, Moscow, 1999: 206-217

Haff G, Haff E. Training integration and periodization - NSCA's program design. Human Kinetics, Champaign IL; 2012

Harre D. Principles of sports training. Sportverlag, Berlin, 1982: 231

Issurin V. Block periodization. Ultimate Athlete Concepts; 2008

Keller W. System of sport preparation. SAAM Moscow, 1995: 41-50

Kostikowa L, Wolosowicz A. Technology of sports training. Basketball, Moscow, 1997: 305-363

Kraemer W. Roundtable discussion: periodization of training (part 1). Strength and Conditioning Journal, 2004; 26(1): 50-53

Liszczenko WE. How to build a long-term training program for elite athletes. Theory and Practice of Physical Culture, 1997; 3: 21-22

Liszczenko WE. Classical periodization and its variants in modern sport. International Congress "Nations health: systems of life long Physical education as a foundation of public health". Moscow, 2014: 98-105 
Liszczenko W. Training periodization of elite tennis players. International Congress „Nations health systems of life long physical education as a foundation of public health". Moscow, 2014: 94-98

Lyakh W. Basketball. AWF, Kraków; 2003

Maglischo E. Swimming fastest. Human Kinetics Champaign IL; 2003

Malacko J. Technology of sports training. Odoja; 2004

Matwiejew LP. The problem of sport training periodization. Physical Culture and Sport, 1964

Matwiejew LP. Fundamentals of sports training. Physical Culture and Sport, 1977

Matwiejew LP. Planning of sports training. Theory and Practice of Physical Culture, 1991; 12: 11-20

Matwiejew LP. Theory of sport. Moscow; 1997

Matwiejew LP. Fundamentals of sports preparation. Olimpic Literature, 1999

Mikolajec K, Maszczyk A, Zajac T. Game Indicators Determining Sports Performance in the NBA. Journal of Human Kinetics, 2013; 37: 145-151.

Nadori L, Granek I. Theoretical and methodological basis of training planning with special considerations within a macrocycle. NSCA Lincoln, NE; 1989

O'Bryant H. Rountable discussion: periodization of training (part 1). Strenght and Conditioning Journal, 2004; (26) 1

Ozolin NG. Modern sports training. Physical Culture and Sport, 1970

Pfeifer H. Fundamentals and principles of endurance training. H. Pfeifer, D. Harre. Principles of Sports Training. - Berlin: Sportverlag, 108-124; 1982

Platonov V. Training of highly skilled athletes. Physical Culture and Sport, 1986

Platonov V. General theory of sports preparation in Olympic Disciplines. Olimpic Literature, 1997

Platonov V. Periodization concepts of sports training. Theory and Practice of Physical Culture, 1998; 8: 23-26, 3946

Platonov V. System of sports preparation in Olympic Disciplines. Olimpic Literature, 2004

Platonov V. The Basis of Modern Training Process Periodization in High-Performance Athletes for Year Preparation. Research Yearbook. National University of Ukraine on Physical Education and Sport, 2006; 12(2): 176-180

Platonow V. Theory of annual training periodization. Olympic Sport Science, 2008; 1: 3-23

Platonov V. Sports training peridization. General Theory and its Practical Application. Olimpic Literature, Kiev; 2013

Stone M. Roundtable discussion: periodization of training (part 1). Strength and Conditioning Journal, 2004; 26(1): 58-60

Stone MH, Stone ME, Sands W. Principles and Practice of Resistance training. Human Kinetics, Champaign IL; 2007

Suslow FP. Modern training. SAAM, 1995: 73-79

Suslow FP. Modern training in individual sports during Olympic preparation. Theory and Practice of Physical Culture, 2002; 11: 30-33

Suslow FP, Filin VP. Important crisis of modern sports theory? Theory and Practice of Physical Culture, 1998; 6: 50-53

Wathen D. Periodization: concepts and applications. Human Kinetics Champaign IL, 507-522; 2008

Verhoshansky Y. Planning and organization of training. Theory and Practice of Physical Culture, 1985

Verhoshansky Y. On the path of training process. Theory and Practice of Physical Culture, 1998; 2: 21-27

(C) Editorial Committee of Journal of Human Kinetics 
Verhoshansky Y. Theory and methodology of sports training. Theory and Practice of Physical Culture, 1998; 7: 41-54

Verhoshansky Y. Theory and methodology of sports training: block training system for elite athletes. Theory and Practice of Physical Culture, 2005: 2-14

\section{Corresponding author:}

\section{Mikołajec Kazimierz}

Department of Team Sport Games,

Jerzy Kukuczka Academy of Physical Education in Katowice,

Mikolowska 72A, 40-065 Katowice, Poland.

Phone: +48727444444

E-mail: k.mikolajec@awf.katowice.pl 